PROCEEDINGS OF THE

AMERICAN MATHEMATICAL SOCIETY

Volume 130, Number 5, Pages 1477-1485

S 0002-9939(01)06235-9

Article electronically published on October 12, 2001

\title{
DIRECTIVE TREES AND GAMES ON POSETS
}

\author{
TETSUYA ISHIU AND YASUO YOSHINOBU
}

(Communicated by Carl G. Jockusch, Jr.)

\begin{abstract}
We show that for any infinite cardinal $\kappa$, every $(\kappa+1)$-strategically closed poset is $\kappa^{+}$-strategically closed if and only if $\square_{\kappa}$ holds. This extends previous results of Velleman, et.al.
\end{abstract}

\section{INTRODUCTION}

In this paper we study a property of posets called 'strategic closure', characterized in terms of games on posets, which have been studied by Jech [J1], [J2], Foreman $[\mathrm{F}]$, Veličkovič $\overline{\mathrm{Vc}}$, Velleman $\mathrm{Vm}$, etc.

Throughout this paper, we count 0 as a successor ordinal, not as a limit ordinal, for notational convenience.

Our notation is based on that of $[\mathrm{F}$, but slightly modified.

Definition 1.1. For a poset $\mathbb{P}$ and an ordinal $\alpha>\omega$, we denote the following twoplayer game as $G_{\alpha}^{\mathrm{I}}(\mathbb{P})$ : At first, Player I chooses $a_{0} \in \mathbb{P}$, Player II chooses $b_{0} \leq_{\mathbb{P}} a_{0}$, and Player I chooses $a_{1} \leq_{\mathbb{P}} b_{0}$. In this manner, both players choose smaller and smaller conditions in turn, say, $b_{1}, a_{2}, b_{2}$, etc. After both have moved $\omega$ times, Player II loses immediately if there is no condition which is smaller than $a_{n}$ for every $n<\omega$. Otherwise, the game continues, as Player I chooses such a condition $a_{\omega}$, Player II chooses $b_{\omega} \leq_{\mathbb{P}} a_{\omega}$, and so on. Other limit stages are played similarly. Player II wins if she could move $\alpha$ times (note that the $\alpha$-th move is not necessary). Otherwise Player I wins.

$G_{\alpha}^{\mathrm{II}}(\mathbb{P})$ denotes the same game but Player II goes first at limit stages.

$$
\begin{array}{lrllllllllll}
G_{\alpha}^{\mathrm{I}}(\mathbb{P}) & \text { I } & : & a_{0} & & a_{1} & & \cdots & a_{\omega} & & a_{\omega+1} & \cdots \\
\text { II } & : & & b_{0} & & b_{1} & \cdots & & b_{\omega} & & \cdots \\
& & & & & & & & & & & \\
G_{\alpha}^{\mathrm{II}}(\mathbb{P}) & \text { I } & : & a_{0} & & a_{1} & & \cdots & & a_{\omega+1} & & \cdots \\
& \text { II } & : & & b_{0} & & b_{1} & \cdots & b_{\omega} & & b_{\omega+1} & \cdots
\end{array}
$$

Note that $G_{\omega+1}^{\mathrm{I}}(\mathbb{P})$ and $G_{\omega+1}^{\mathrm{II}}(\mathbb{P})$ are essentially the same game.

Received by the editors November 2, 2000.

2000 Mathematics Subject Classification. Primary 03E40; Secondary 03E65.

Key words and phrases. Directive trees, strategic closure.

The second author was partially supported by Grant-in-Aid for Scientific Research (No.11640112), Ministry of Education, Science and Culture, Japan.

(C)2001 American Mathematical Society 
We reserve the notation $a_{\beta}$ and $b_{\beta}$ to denote Player I's and Player II's $\beta$-th move in $G^{\mathrm{I}}$-type games. We also use a similar notation in $G^{\mathrm{II}}$-type games, but in these games we let Player I 'skip' the limit lower cases, to simplify our notation.

Definition 1.2. Let $\alpha>\omega$ and let $\mathbb{P}$ be a poset. A strategy (for Player II) in $G_{\alpha}^{\mathrm{I}}(\mathbb{P})$ is a function of the form

$$
\sigma: \bigcup_{\beta<\alpha}^{\beta+1} \mathbb{P} \rightarrow \mathbb{P}
$$

Player II is said to play by a strategy $\sigma$ if she chooses $\sigma\left(\left\langle a_{\gamma} \mid \gamma \leq \beta\right\rangle\right)$ as $b_{\beta}$, as long as she survives. A strategy $\sigma$ is a winning one if Player II wins whenever she plays by $\sigma$.

Similarly, a strategy (for Player II) in $G_{\alpha}^{\mathrm{II}}(\mathbb{P})$ is of the form

$$
\sigma: \bigcup_{\beta<\alpha}{ }^{1+\beta} \mathbb{P} \rightarrow \mathbb{P}
$$

Player II plays by $\sigma$ if she chooses $\sigma\left(\left\langle a_{\gamma}\right| \gamma \leq \beta\right.$ and $\gamma$ is a successor $\left.\rangle\right)$ as $b_{\beta}$, and so on.

Of course strategies for Player I can be defined similarly, but we will not use them in this paper. Concerning them, instead, we just mention the following remarkable result.

Theorem 1.3 (Banach-Mazur, Jech). A poset $\mathbb{P}$ is $\sigma$-Baire iff Player I does not have a winning strategy in $G_{\omega+1}^{\mathrm{I}(\mathrm{II})}(\mathbb{P})$.

Definition 1.4. For a poset $\mathbb{P}$ and an ordinal $\alpha>\omega, \mathbb{P}$ is $\alpha$-strategically closed (resp. strongly $\alpha$-strategically closed) if Player II has a winning strategy for $G_{\alpha}^{\mathrm{II}}(\mathbb{P})$ $\left(\right.$ resp. $\left.G_{\alpha}^{\mathrm{I}}(\mathbb{P})\right)$.

Note that the term 'strong' is valid, since if Player II has a winning strategy for a $G^{\mathrm{I}}$-type game, then she can win the corresponding $G^{\mathrm{II}}$-type game by considering 'imaginary moves' of Player I at limit stages.

In this paper we discuss when a shorter strategic closure implies a longer strategic closure. Lemma 2.2, Lemma 2.3 for $\lambda=\omega$, and Proposition 3.5 are due to the first author, whereas Lemma 2.3 in general, and Theorem 3.3 are due to the second. Some results of the first author are also described in his master's thesis []].

Here we introduce another type of game first considered by Velleman [Vm].

Definition 1.5. For a poset $\mathbb{P}$ and a limit ordinal $\alpha$, we denote the following twoplayer game as $G_{\alpha}^{V}(\mathbb{P})$ : Players construct a sequence $\left\langle a_{\beta} \mid \beta<\alpha\right\rangle$ consisting of descending conditions of $\mathbb{P}$. Player I moves at every successor stage (including stage 0), and Player II at every limit stage. Player I wins if Player II is unable to choose a legal move at some stage. Otherwise, Player II wins.

In fact, Velleman's games are closely related to $G^{\mathrm{II}}$-type games by the following fact.

Proposition 1.6. For a poset $\mathbb{P}$ and an ordinal $\alpha>\omega$, Player II has a winning strategy for $G_{\omega \alpha}^{V}(\mathbb{P})$ iff $\mathbb{P}$ is $\sigma$-closed and $\alpha$-strategically closed.

Proof. Suppose Player II has a winning strategy for $G_{\omega \alpha}^{V}(\mathbb{P})$. This means the game never stops at stage $\omega$, no matter how Player I moves. This shows that $\mathbb{P}$ is $\sigma$ closed. Moreover, Player II can win $G_{\alpha}^{\mathrm{II}}(\mathbb{P})$ by considering each move of Player I 
as $\omega$ successive moves consisting of the same conditions as in the game $G_{\omega \alpha}^{V}(\mathbb{P})$ and applying the winning strategy for $G_{\omega \alpha}^{V}(\mathbb{P})$. Conversely, suppose $\mathbb{P}$ is $\sigma$-closed and $\alpha$-strategically closed. Since $\mathbb{P}$ is $\sigma$-closed, whenever Player I moves $\omega$ times successively in the game $G_{\omega \alpha}^{V}(\mathbb{P})$, one can pick an even smaller condition. Thus, considering it as Player I's move in the game $G_{\alpha}^{\mathrm{II}}(\mathbb{P})$ and applying the winning strategy for $G_{\alpha}^{\mathrm{II}}(\mathbb{P})$ makes Player II win the game $G_{\omega \alpha}^{V}(\mathbb{P})$.

\section{Directive trees}

In this section, we introduce the notion of directive trees, a key tool for our construction of 'longer' winning strategies.

Definition 2.1. Let $\lambda$ be an ordinal, and $\kappa$ a cardinal. A tree $T=(\lambda, \prec)$ is called $(\lambda, \kappa)$-directive if the following conditions hold:

(1) height $(T) \leq \kappa$, where height $(T)$ denotes the height of $T$ as a tree.

(2) $\forall \alpha, \beta<\lambda[\alpha \prec \beta \Rightarrow \alpha<\beta]$.

(3) For every limit ordinal $\eta \leq \lambda$ such that $\mathrm{cf} \eta \leq \kappa$, there is a branch $b$ of $T$ (of limit length) such that $\sup b=\eta$.

A $(\lambda, \kappa)$-directive tree $T$ is called continuous if every branch of $T$ is continuous as a sequence of ordinals.

The following is a key lemma for our main theorem:

Lemma 2.2. Let $\kappa$ be an infinite cardinal.

(1) If there is a $\left(\kappa^{+}, \kappa\right)$-directive tree, then every strongly $(\kappa+1)$-strategically closed poset is strongly $\kappa^{+}$-strategically closed.

(2) If there is a continuous $\left(\kappa^{+}, \kappa\right)$-directive tree, then every $(\kappa+1)$-strategically closed poset is $\kappa^{+}$-strategically closed.

Proof. (1) Let $T$ be a $\left(\kappa^{+}, \kappa\right)$-directive tree, and $\sigma$ a winning strategy for $G_{\kappa+1}^{\mathrm{I}}(\mathbb{P})$. We will construct a strategy $\tau$ for $G_{\kappa^{+}}^{\mathrm{I}}(\mathbb{P})$. For each ordinal $\beta<\kappa^{+}$, let $\left\langle\beta_{\xi} \mid \xi \leq \eta\right\rangle$ be the unique branch of $T$ such that $\beta_{\eta}=\beta$. For each $\left\langle a_{\gamma} \mid \gamma \leq \beta\right\rangle \in{ }^{\beta+1} \mathbb{P}$ we define

$$
\tau\left(\left\langle a_{\gamma} \mid \gamma \leq \beta\right\rangle\right):=\sigma\left(\left\langle a_{\beta_{\xi}} \mid \xi \leq \eta\right\rangle\right) .
$$

Now let us check that $\tau$ is a winning strategy. Suppose Player II plays by $\tau$ in $G_{\kappa^{+}}^{\mathrm{I}}(\mathbb{P})$. By induction on $\beta<\kappa^{+}$, we will show that Player II can make her $\beta$-th move legally. By the induction hypothesis, we may assume that both players have moved $\beta$ times legally.

If $\beta$ is a successor, we may also assume Player I could choose his next move $a_{\beta}$. Then suppose $\beta$ is limit. By (3) of Definition 2.1, there is a branch $b$ of $T$ (of limit length) such that $\sup b=\beta$. Let $\left\langle\beta_{\xi}^{\prime} \mid \xi<\delta\right\rangle$ be the increasing enumeration of $b$ (thus $\delta$ is a limit ordinal $\leq \kappa$ ). For each $\xi<\delta$, by our assumption,

$$
b_{\beta_{\xi}^{\prime}}=\tau\left(\left\langle a_{\gamma} \mid \gamma \leq \beta_{\xi}^{\prime}\right\rangle\right)=\sigma\left(\left\langle a_{\beta_{\zeta}^{\prime}} \mid \zeta \leq \xi\right\rangle\right)
$$

holds. This shows that $\left\langle a_{\beta_{\xi}^{\prime}}, b_{\beta_{\xi}^{\prime}} \mid \xi<\delta\right\rangle$ forms a record of $G_{\kappa+1}^{\mathrm{I}}(\mathbb{P})$, in which Player II played by $\sigma$. Since $\sigma$ is a winning strategy for $G_{\kappa+1}^{\mathrm{I}}(\mathbb{P}),\left\langle a_{\beta_{\xi}^{\prime}} \mid \xi<\delta\right\rangle$ has a common extension in $\mathbb{P}$. So does $\left\langle a_{\gamma}, b_{\gamma} \mid \gamma<\beta\right\rangle$, since it is decreasing and $b$ is cofinal in $\beta$. Therefore we may assume that Player I could choose $a_{\beta}$ even if $\beta$ is limit. 
Now let $\left\langle\beta_{\xi} \mid \xi \leq \eta\right\rangle$ be the unique branch of $T$ such that $\beta_{\eta}=\beta$. By the same argument as above, $\left\langle a_{\beta_{\xi}}, b_{\beta_{\xi}} \mid \xi<\eta\right\rangle^{\wedge}\left\langle a_{\beta}\right\rangle$ forms a record of the game $G_{\kappa+1}^{\mathrm{I}}(\mathbb{P})$, in which Player II plays by $\sigma$. Therefore, since $\sigma$ is winning,

$$
b_{\beta}=\tau\left(\left\langle a_{\gamma} \mid \gamma \leq \beta\right\rangle\right)=\sigma\left(\left\langle a_{\beta_{\xi}} \mid \xi \leq \eta\right\rangle\right) \leq a_{\beta_{\eta}}=a_{\beta}
$$

holds. This shows Player II can make her $\beta$-th move legally, and the induction is completed.

(2) Let $T$ be a continuous $\left(\kappa^{+}, \kappa\right)$-directive tree and $\sigma$ a winning strategy for $G_{\kappa+1}^{\mathrm{II}}(\mathbb{P})$. We set

$$
\begin{aligned}
S & :=\left\{\beta<\kappa^{+} \mid \beta \text { is a successor }\right\}, \\
L_{1} & :=\left\{\beta<\kappa^{+} \mid \beta \text { is limit and at a successor level of } T\right\}, \text { and } \\
L_{2} & :=\left\{\beta<\kappa^{+} \mid \beta \text { is limit and at a limit level of } T\right\} .
\end{aligned}
$$

Note that by the continuity of $T$, every successor ordinal $\beta<\kappa^{+}$is placed at a successor level of $T$.

Fix a function

$$
F: \bigcup_{\beta<\kappa^{+}, \operatorname{Lim}(\beta)} \beta \mathbb{P} \rightarrow \mathbb{P}
$$

such that for every $s$ in its domain, $F(s) \in \mathbb{P}$ is smaller than every condition occurring in $s$, if such a condition exists.

Every element of ${ }^{1+\beta} \mathbb{P}\left(\beta<\kappa^{+}\right)$can be written as $\left\langle a_{\gamma}\right| \gamma \leq \beta, \gamma$ is a successor $\rangle$. Under this notation, we 'fill up the skipped lower cases', that is, for each limit ordinal $\delta \leq \beta$, we define

$$
\left.a_{\delta}:=F\left(\left\langle a_{\gamma}\right| \gamma<\delta, \gamma \text { is a successor }\right\rangle\right) .
$$

For $\beta<\kappa^{+}$, let $\left\langle\beta_{\xi} \mid \xi \leq \eta\right\rangle$ be the unique branch of $T$ such that $\beta_{\eta}=\beta$. Now we define $\tau$ on ${ }^{1+\beta} \mathbb{P}$ as follows:

$$
\left.\left.\tau\left(\left\langle a_{\gamma}\right| \gamma \leq \beta, \gamma \text { is a successor }\right\rangle\right):=\sigma\left(\left\langle a_{\beta_{\xi}}\right| \xi \leq \eta, \xi \text { is a successor }\right\rangle\right) .
$$

Now we suppose that Player II plays by $\tau$, and that Player I plays legally as long as possible. By induction on $\beta<\kappa^{+}$, we show that

(i) Player II can make her $\beta$-th move $b_{\beta}$ legally, and

(ii) $\left\langle a_{\gamma} \mid \gamma \leq \beta, \gamma \in S \cup L_{1}\right\rangle$ is decreasing.

Case 1. $\beta \in S$.

By the induction hypothesis, we may assume that Player II has played her first $\beta$ turns, and thereafter Player I chose $a_{\beta}$ legally. This and (ii) of the induction hypothesis imply (ii) of this stage. Let $\left\langle\beta_{\xi} \mid \xi \leq \eta\right\rangle$ be the unique branch of $T$ such that $\beta_{\eta}=\beta$. For each $\xi<\eta$, by our assumption,

$$
\begin{aligned}
b_{\beta_{\xi}} & \left.=\tau\left(\left\langle a_{\gamma}\right| \gamma \leq \beta_{\xi}, \gamma \text { is a successor }\right\rangle\right) \\
& \left.=\sigma\left(\left\langle a_{\beta_{\zeta}}\right| \zeta \leq \xi, \zeta \text { is a successor }\right\rangle\right)
\end{aligned}
$$

holds. Moreover, for each $\xi<\eta, b_{\beta_{\xi}} \geq a_{\beta_{\xi}+1} \geq a_{\beta_{\xi+1}}$ holds (the second inequality is derived from (ii) of the induction hypothesis). These facts assure that

$$
\left.\left\langle\left\langle a_{\beta_{\xi}}\right| \xi \leq \eta, \xi \text { is a successor }\right\rangle,\left\langle b_{\beta_{\xi}} \mid \xi<\eta\right\rangle\right\rangle
$$


forms a record of the game $G_{\kappa+1}^{\mathrm{II}}(\mathbb{P})$ in which Player II plays by $\sigma$. Since $\sigma$ is winning,

$$
\left.b_{\beta}=\sigma\left(\left\langle a_{\beta_{\xi}}\right| \xi \leq \eta, \xi \text { is a successor }\right\rangle\right) \leq a_{\beta_{\eta}}=a_{\beta}
$$

holds. This shows (i) of this stage.

Case 2. $\beta \in L_{1}$.

Let $b=\left\langle\beta_{\xi}^{\prime} \mid \xi<\delta\right\rangle$ be a branch of $T$ such that $\delta$ is a limit ordinal $\leq \kappa$ and $\sup b=\beta$. By the same argument as in Case 1 ,

$$
\left.\left\langle\left\langle a_{\beta_{\xi}^{\prime}}\right| \xi<\delta, \xi \text { is a successor }\right\rangle,\left\langle b_{\beta_{\xi}^{\prime}} \mid \xi<\delta\right\rangle\right\rangle
$$

forms a record of $G_{\kappa+1}^{\mathrm{II}}(\mathbb{P})$, in which Player II plays by $\sigma$, and since $\sigma$ is winning, $\left\langle a_{\beta_{\xi}^{\prime}}\right| \xi<\delta, \xi$ is a successor $\rangle$ has a common extension. Since $b$ is cofinal in $\beta$, by (ii) of the induction hypothesis, $\left\langle a_{\gamma} \mid \gamma<\beta, \gamma \in S \cup L_{1}\right\rangle$ also has a common extension, and therefore by $\left(^{*}\right)$ and the definition of $F, a_{\beta}$ is such a common extension. This shows (ii) of this stage.

Now letting $\left\langle\beta_{\xi} \mid \xi \leq \eta\right\rangle$ be the unique branch of $T$ such that $\beta_{\eta}=\beta$, (i) can be proved in the same way as Case 1 .

Case 3. $\beta \in L_{2}$.

We have nothing to do for (ii). For (i), let $\left\langle\beta_{\xi} \mid \xi \leq \eta\right\rangle$ be the unique branch of $T$ such that $\beta_{\eta}=\beta$. Since $\beta \in L_{2}, \eta$ is a limit ordinal, and by the continuity of $T$, $\left\langle\beta_{\xi} \mid \xi<\eta\right\rangle$ is cofinal in $\beta$. By the same argument as above,

$$
\left.\left\langle\left\langle a_{\beta_{\xi}}\right| \xi<\eta, \xi \text { is a successor }\right\rangle,\left\langle b_{\beta_{\xi}} \mid \xi<\eta\right\rangle\right\rangle
$$

forms a record of $G_{\kappa+1}^{\mathrm{II}}(\mathbb{P})$, in which Player II plays by $\sigma$, and since $\sigma$ is winning,

$$
\left.b_{\beta}=\sigma\left(\left\langle a_{\beta_{\xi}}\right| \xi<\eta, \xi \text { is a successor }\right\rangle\right)
$$

is a common extension of $\left\langle a_{\beta_{\xi}}\right| \xi<\eta, \xi$ is a successor $\rangle$, and thus of $\left\langle a_{\gamma}\right| \gamma<$ $\beta, \gamma$ is a successor $\rangle$, since this is decreasing by (ii) of the induction hypothesis and $\left\langle\beta_{\xi} \mid \xi<\eta\right\rangle$ is cofinal in $\beta$. This proves (i).

The induction is completed, and thus $\tau$ is shown to be a winning strategy.

The following lemma will be used later.

Lemma 2.3. For every infinite cardinal $\lambda$, there is a $(\lambda, \omega)$-directive tree.

Proof. By induction on $\lambda$, we will construct a $(\lambda, \omega)$-directive tree $\langle\lambda, \prec \mid \lambda\rangle$ as a subtree of a 'global' tree $\langle$ Ord, $\prec\rangle$. For $\lambda=\omega$, let $n+1$ be a $\prec$-immediate successor of $n$ for each $n \in \omega$. It is clear that $\langle\omega, \prec \mid \omega\rangle$ forms an $(\omega, \omega)$-directive tree.

Now suppose $\langle\lambda, \prec \mid \lambda\rangle$ is defined to be $(\lambda, \omega)$-directive. We will extend this to a tree on $\lambda^{+}$. For $\alpha<\lambda^{+}$, put

$$
\begin{aligned}
& E_{\alpha}:=\{\beta \in[\lambda \alpha, \lambda \alpha+\lambda) \mid \beta \text { is even }\}, \\
& O_{\alpha}:=\{\beta \in[\lambda \alpha, \lambda \alpha+\lambda) \mid \beta \text { is odd }\} .
\end{aligned}
$$

Our construction goes as follows:

(1) For each $\alpha \in\left(0, \lambda^{+}\right)$, let $\prec \uparrow O_{\alpha}$ be the isomorphic copy of $\prec \uparrow \lambda$ under the order isomorphism between $\lambda$ and $O_{\alpha}$. Each $O_{\alpha}$ will be independent from the other part of our final tree on $\lambda^{+}$.

(2) By induction on $\alpha<\lambda^{+}$, we extend $\langle\lambda, \prec \uparrow \lambda\rangle$ to $S_{\alpha}=\lambda \cup \bigcup_{\gamma<\alpha} E_{\gamma}$ as follows: Assume $\left\langle S_{\alpha}, \prec \mid S_{\alpha}\right\rangle$ is already defined. Fix a bijection $g_{\alpha}: E_{\alpha} \rightarrow S_{\alpha}$ and let each 
$\beta \in E_{\alpha}$ be a $\prec$-immediate successor of $g_{\alpha}(\beta)$. This defines $\left\langle S_{\alpha+1} \prec \nmid S_{\alpha+1}\right\rangle$. At limit stages, we just take the union of predecessors.

Now let us check that $\left\langle\lambda^{+}, \prec \mid \lambda^{+}\right\rangle$is $\left(\lambda^{+}, \omega\right)$-directive. Note that $\left\langle\lambda^{+}, \prec \mid \lambda^{+}\right\rangle$is a tree of height $\omega$, since the new odd ordinals form trees isomorphic to $\langle\lambda, \prec \mid \lambda\rangle$, whereas each new even ordinal is set as an immediate successor of an element of finite level. (1) and (2) of Definition 2.1 are clear. We will show that (3) holds. Let $\alpha<\lambda^{+}$be such that $\operatorname{cf}(\alpha)=\omega$.

Case 1. $\alpha$ is not a multiple of $\lambda \omega$.

In this case $\alpha$ can be written as $\lambda \beta+\xi$, where $\xi \leq \lambda$ and $\operatorname{cf}(\xi)=\omega$. Since the order isomorphism between $\lambda$ and $O_{\beta}$ maps any strictly increasing sequence converging to $\xi$ to one converging to $\lambda \beta+\xi=\alpha$, we have a branch $b$ of $O_{\beta}$ such that $\sup b=\alpha$.

Case 2. $\alpha$ is a multiple of $\lambda \omega$.

Let $\left\langle\lambda \alpha_{n} \mid n<\omega\right\rangle$ be a strictly increasing sequence of multiples of $\lambda$, converging to $\alpha$. Now let $\beta_{0}=0$ and $\beta_{n+1}=g_{\alpha_{n}}{ }^{-1}\left(\beta_{n}\right)$. Then for every $n<\omega, \beta_{n+1}$ is a $\prec$-immediate successor of $\beta_{n}$, and $\lambda \alpha_{n} \leq \beta_{n+1}<\lambda \alpha_{n}+\lambda \leq \lambda \alpha_{n+1}$ holds. This shows that $b=\left\langle\beta_{n}\right| n\langle\omega\rangle$ is a branch of $\left\langle\lambda^{+}, \prec \uparrow \lambda^{+}\right\rangle$and converges to $\alpha$. This finishes the proof that $\left\langle\lambda^{+}, \prec \mid \lambda^{+}\right\rangle$is $\left(\lambda^{+}, \omega\right)$-directive.

For a limit cardinal $\lambda$, we let $\prec \uparrow \lambda=\bigcup_{\delta<\lambda}(\prec \uparrow \delta)$. The induction hypothesis assures almost all conditions for $\langle\lambda, \prec \mid \lambda\rangle$ to be a $(\lambda, \omega)$-directive tree, except the existence of a branch converging to $\lambda$ (this is needed only in the case $\operatorname{cf} \lambda=\omega$ ), which can be seen just as in the proof of Case 2 above.

\section{MAIN THEOREM}

In this section we state our main theorem on the prolongation of the strategic closure property, and give its proof.

As a preceding result of this kind, Velleman $[\mathrm{Vm}]$ showed the following concerning his games.

Theorem 3.1 (Velleman). Let $\kappa$ be an infinite cardinal. Then the following are equivalent:

(1) $\square_{\kappa}$.

(2) For every poset $\mathbb{P}$, if Player II has a winning strategy for $G_{\kappa+\omega}^{V}(\mathbb{P})$, then Player II also has a winning strategy for $G_{\kappa^{+}}^{V}(\mathbb{P})$.

According to Proposition 1.6, this theorem says that $\square_{\kappa}$ is equivalent to the principle that $(\kappa+1)$-strategic closure implies $\kappa^{+}$-strategic closure for $\sigma$-closed posets.

Note that this theorem says almost nothing in the case $\kappa=\omega$, since Player II trivially wins in games of length $\omega_{1}$ on any $\sigma$-closed posets.

On the other hand, Lemma 2.3 for $\lambda=\omega_{1}$ gives the following, which is known also by Foreman and Veličkovič independently (see [I], [J2] and [Vc]).

Corollary 3.2 (Foreman, Veličkovič). Every $(\omega+1)$-strategically closed poset is strongly $\omega_{1}$-strategically closed.

Remark. Thus for $\alpha \leq \omega_{1}$, the two notions of strategic closure are equivalent. It is shown by Gray [G] that there is a poset which is $\left(\omega_{1}+1\right)$-strategically closed but not strongly $\left(\omega_{1}+1\right)$-strategically closed (see Foreman $[\mathbf{F}]$ ). 
Our main theorem, which can be viewed as the unification of these two theorems, is the following.

Theorem 3.3. Let $\kappa$ be an infinite cardinal. Then the following are equivalent:

(1) $\square_{\kappa}$.

(2) There is a continuous $\left(\kappa^{+}, \kappa\right)$-directive tree.

(3) Every $(\kappa+1)$-strategically closed poset is $\kappa^{+}$-strategically closed.

Proof. Note that $(2) \Rightarrow(3)$ is Lemma $2.2(2)$, and that $(3) \Rightarrow(1)$ is immediate by Theorem 3.1 and Proposition 1.6. Thus it is enough to show that (1) implies (2).

Suppose $\left\langle C_{\alpha}\right| \alpha<\kappa^{+}, \alpha$ is a limit $\rangle$ is a $\square_{\kappa}$-sequence, that is, each $C_{\alpha}$ satisfies the following conditions:

1. $C_{\alpha}$ is a club subset of $\alpha$.

2. o.t. $\left(C_{\alpha}\right) \leq \kappa$.

3. If $\beta<\alpha$ is a limit point of $C_{\alpha}, C_{\alpha} \cap \beta=C_{\beta}$ holds. Let

$$
C:=\bigcup_{\alpha<\kappa^{+}, \operatorname{Lim}(\alpha)} \text { l.p. }\left(C_{\alpha}\right) \cap \alpha,
$$

where l.p. $\left(C_{\alpha}\right)$ denotes the set of limit points of $C_{\alpha}$. We construct an auxiliary tree $\left\langle C, \prec_{0}\right\rangle$, as follows:

$$
\beta \prec_{0} \gamma \underset{\text { def }}{\Leftrightarrow} \beta \in \text { l.p. }\left(C_{\gamma}\right) \cap \gamma .
$$

Whenever $\beta, \gamma \in C$ and $\beta \prec_{0} \gamma, C_{\beta}=C_{\gamma} \cap \beta$ holds, and therefore $\xi \prec_{0} \beta$ is equivalent to $\xi \prec_{0} \gamma$ for $\xi \in C \cap \beta$. This assures that $\prec_{0}$ is transitive and that the initial segment of $\left\langle C, \prec_{0}\right\rangle$ by any member of $C$ is well-ordered by $\prec_{0}$. Therefore, $\left\langle C, \prec_{0}\right\rangle$ is a tree. By (2) of the definition of $\square$-sequence, the height of this tree is $\leq \kappa$. Moreover, for every limit ordinal $\alpha<\kappa^{+}$such that $\operatorname{cf}(\alpha)>\omega$, l.p. $\left(C_{\alpha}\right) \cap \alpha$ forms a normal branch of $\left\langle C, \prec_{0}\right\rangle$, converging to $\alpha$.

On the other hand, by Lemma 2.3 , we have a $\left(\kappa^{+}, \omega\right)$-directive tree $\left\langle\kappa^{+}, \prec_{1}\right\rangle$, which has branches (of length $\omega$ ) converging to every ordinal $\left(<\kappa^{+}\right)$of cofinality $\omega$. We 'mix' these two trees to get a final tree.

Next let $\left\langle S, \prec_{2}\right\rangle$ be the isomorphic copy of $\left\langle\kappa^{+}, \prec_{1}\right\rangle$ transformed by the order isomorphism between $\kappa^{+}$and $S$, the set of the successor ordinals less than $\kappa^{+}$. Since this order isomorphism does not change the supremum of any $\omega$-increasing sequence of ordinals less than $\kappa^{+},\left\langle S, \prec_{2}\right\rangle$ also has branches (of length $\omega$ ) converging to every ordinal $\left(<\kappa^{+}\right)$of cofinality $\omega$.

Now let $\prec=\prec_{0} \cup \prec_{2}$. Since $C$ consists only of limit ordinals and thus does not intersect $S, \prec$ is a tree ordering, and now it is easy to see that $\left\langle\kappa^{+}, \prec\right\rangle$ satisfies all conditions to be a continuous $\left(\kappa^{+}, \kappa\right)$-directive tree.

Note that the same argument gives the following, because a 'partial $\square_{\kappa}$-sequence' of length $\gamma$ and thus a continuous $(\gamma, \kappa)$-directive tree always exists for arbitrary $\gamma<\kappa^{+}$.

Corollary 3.4. Suppose $\kappa$ is an infinite cardinal. Then every (resp. strongly) ( $\kappa+$ 1)-strategically closed poset is (resp. strongly) $\gamma$-strategically closed for arbitrary $\gamma<\kappa^{+}$.

Question. To what is the existence of a $\left(\kappa^{+}, \kappa\right)$-directive tree or 'strong $(\kappa+1)$ strategic closure implies strong $\kappa^{+}$-strategic closure' (for $\kappa$ uncountable) equivalent? 
Of course, $\square_{\kappa}$ is a sufficient condition by Lemma 2.2 and Theorem 3.3. Another sufficient condition is given by the following:

Proposition 3.5. If $\kappa^{<\kappa}=\kappa$ holds, then there is a $\left(\kappa^{+}, \kappa\right)$-directive tree.

By induction on $\alpha \leq \kappa^{+}$, we will construct a $(\kappa \alpha, \kappa)$-directive tree $\langle\kappa \alpha, \prec \mid \kappa \alpha\rangle$ as a subtree of $\left\langle\kappa^{+}, \prec\right\rangle$, which is our final tree. For $\alpha=0$, we have nothing to do, and for $\alpha=1$, we let $\prec \mid \kappa=\langle\uparrow \kappa$. Clearly $\langle\kappa, \prec \mid \kappa\rangle$ is a $(\kappa, \kappa)$-directive tree.

Now suppose that $0<\alpha<\kappa^{+}$and $\langle\kappa \alpha, \prec \uparrow \kappa \alpha\rangle$ is defined to be a $(\kappa \alpha, \kappa)$-directive tree. Let

$$
\begin{aligned}
& E_{\alpha}=\{\beta \in[\kappa \alpha, \kappa(\alpha+1)) \mid \beta \text { is even }\}, \\
& O_{\alpha}=\{\beta \in[\kappa \alpha, \kappa(\alpha+1)) \mid \beta \text { is odd }\}, \text { and } \\
& B_{\alpha}=\{b \subseteq \kappa \alpha \mid b \text { is a branch of }\langle\kappa \alpha, \prec \mid \kappa \alpha\rangle \wedge|b|<\kappa\} .
\end{aligned}
$$

Note that $\left|B_{\alpha}\right|=\kappa$ since $|\kappa \alpha|=\kappa$ and $\kappa^{<\kappa}=\kappa$. Thus we can pick a bijection $f_{\alpha}: E_{\alpha} \rightarrow B_{\alpha}$. We extend $\langle\kappa \alpha, \prec \uparrow \kappa \alpha\rangle$ to $\langle\kappa(\alpha+1), \prec \uparrow \kappa(\alpha+1)\rangle$ as follows: First add each $\xi \in E_{\alpha}$ to $\langle\kappa \alpha, \prec \mid \kappa \alpha\rangle$ as an immediate successor of the branch $f_{\alpha}(\xi)$. Then let $O_{\alpha}$ be an independent branch which is naturally ordered.

Let us check that $\langle\kappa(\alpha+1), \prec \mid \kappa(\alpha+1)\rangle$ is $\langle\kappa(\alpha+1), \kappa\rangle$-directive. (1) and (2) in Definition 2.1 are clear, and (3) for limit ordinals $\leq \kappa \alpha$ is by the induction hypothesis. For each limit ordinal $\eta$ such that $\kappa \alpha<\eta \leq \kappa(\alpha+1), O_{\alpha} \cap \eta$ forms a branch (of limit length) of $\langle\kappa(\alpha+1), \prec \uparrow(\alpha+1)\rangle$, whose supremum is $\eta$. This proves (3).

For a limit $\alpha<\kappa^{+}$, we just let $\prec \uparrow \kappa \alpha=\bigcup_{\beta<\alpha}(\prec \uparrow \kappa \beta)$. The induction hypothesis implies that $\langle\kappa \alpha, \prec \uparrow \kappa \alpha\rangle$ satisfies all conditions to be $(\kappa \alpha, \kappa)$-directive, except (3) for $\eta=\kappa \alpha$ in Definition 2.1. For this, let $\left\langle\alpha_{i} \mid i<\gamma\right\rangle(\gamma \leq \kappa$ : limit $)$ be a strictly increasing sequence of ordinals converging to $\alpha$. By induction on $i<\gamma$, we define a branch $b=\left\langle\xi_{i} \mid i<\gamma\right\rangle$ such that $b \uparrow i \subseteq \kappa \alpha_{i}$ for each $i<\gamma$, as follows:

Suppose that $b \uparrow i$ is defined and satisfies $b \uparrow i \subseteq \kappa \alpha_{i}$. Let

$$
\xi_{i}:=f_{\alpha_{i}}{ }^{-1}(b \uparrow i) \text {. }
$$

By the definition of $f_{\alpha_{i}}, b \uparrow(i+1)$ is a branch, and

$$
b \uparrow(i+1) \subseteq \kappa \alpha_{i} \cup\left[\kappa \alpha_{i}, \kappa\left(\alpha_{i}+1\right)\right)=\kappa\left(\alpha_{i}+1\right) \subseteq \kappa \alpha_{i+1}
$$

holds. Moreover, suppose that $i<\gamma$ is a limit ordinal, and that $b \uparrow j \subseteq \kappa \alpha_{j}$ holds for every $j<i$. Then $b \uparrow i \subseteq \sup _{j<i} \kappa \alpha_{j} \subseteq \kappa \alpha_{i}$ holds. Thus the induction holds.

Now since $\kappa \alpha_{i} \leq \xi_{i}<\kappa \alpha_{i+1}$ holds for each $i<\gamma$ by the definition, $\sup b=\kappa \alpha$ holds, proving (3).

Finally, we let $\prec=\bigcup_{\alpha<\kappa^{+}}(\prec \uparrow \kappa \alpha)$. In this case the induction hypothesis suffices to show that $\left\langle\kappa^{+}, \prec\right\rangle$ is $\left(\kappa^{+}, \kappa\right)$-directive.

\section{ACKNOWLEDGEMENTS}

The authors would like to express their gratitude to Katsuya Eda, Joel Hamkins, Makoto Takahashi, and Tadatoshi Miyamoto for their helpful comments.

\section{REFERENCES}

[F] M. Foreman, Games played on boolean algebras, J. of Symbolic Logic 48 (1983), 714-723. MR 85h:03064

[G] C. Gray, Iterated forcing from the strategic point of view, Ph.D. Thesis, University of California, Berkeley, 1980. 
[I] T. Ishiu, On Axiom A and games on boolean algebras (Japanese), Master Thesis, Waseda University, 1997.

[J1] T. Jech, A game theoretic property of Boolean algebras, Logic Colloquium '77 (A. Macintyre, L. Pacholski and J. Paris, eds.), North-Holland, Amsterdam, 1978, pp. 135-144. MR 80c:90184

[J2] T. Jech, More game-theoretic properties of Boolean algebras, Ann. Pure Appl. Logic 26 (1984), 11-29. MR 85j:03110

[Vc] B. Veličkovič, Jensen's $\square$ principles and the Novák number of partially ordered sets, J. of Symbolic Logic 51 (1986), 47-58. MR 87h:03074

[Vm] D. Velleman, On a generalization of Jensen's $\square_{\kappa}$, and strategic closure of partial orders, J. of Symbolic Logic 48 (1983), 1046-1052. MR 85g:03076

Department of Mathematics, University of California, Irvine, California 92697

E-mail address: tishiu@math.uci.edu

Graduate School of Human Informatics, Nagoya University, Nagoya 464-8601, Japan

E-mail address: yosinobu@math.nagoya-u.ac.jp 